\title{
Synthesis of Novel pH-responsive PEGylated Zwitterionic Triblock Copolymer
}

\author{
Hai-Liang DONG ${ }^{1, a}$, Zhong-Li NIU ${ }^{1, b}$, Xiao-Ting ZHANG ${ }^{1}$, \\ Shu-Yu ZHU ${ }^{1}$, Ying $\mathrm{YAO}^{1}$, Ya GAO ${ }^{1}$, Bin SUN ${ }^{1}$, Mei-Fang ZHU ${ }^{1}$, \\ Xiao-Ze JIANG ${ }^{1, c}$, \\ ${ }^{1}$ State Key Laboratory for Modification of Chemical Fibers and Polymer Materials, \\ College of Material Science and Engineering, Donghua University, Shanghai, 201620, \\ China \\ aemail: Iddonghailiang@163.com, bemail: nancymiumiu@163.com, email: \\ xiaozejiang@dhu.edu.cn \\ ${ }^{*}$ Corresponding author
}

Keywords: Self-assembly, Triblock copolymer, pH-responsive, ATRP.

\begin{abstract}
Well-defined PEGylated zwitterionic triblock copolymer, poly(ethylene glycol)- $b$-poly(methacrylic acid)- $b$-poly(2-aminoethyl methacrylate) (PEG- $b$-PMAA- $b$ -PAEMA), was prepared from poly(ethylene glycol)-b-poly(tert-butyl meth acrylate) - $b$-poly(N-(tert-butoxycarbonyl) amino ethyl methacrylate)(PEG- $b$-P $t$ BocMA- $b$-P $t$ Boc AEMA) precursor synthesized via atom transer radical polymerization (ATRP) by successively polymerized tert-butyl methacrylate ( $t$ BocMA) and $N$-(tert-butoxycarbonyl) amionethyl methacrylate ( $t$ BocAEMA) monomers using a PEG-based macroinitiator followed by $t$-Boc group deprotection. The obtained triblock copolymer was confirmed by proton nuclear magnetic response $\left({ }^{1} \mathrm{H}\right.$ NMR) and Gel Permeation Chromatography (GPC). The resultant PEG- $b$-PMAA- $b$-PAEMA copolymer bearing a large number of amine moieties will have potential applications as anti-cancer drug or DNA carriers.
\end{abstract}

\section{Introduction}

In the past decades, stimuli double hydrophilic block copolymers (DHBCs) have drawn continuously increasing attentions, for it fantastic self-assembly behaviors, induced by external stimuli such as $\mathrm{pH}$, ionic strength, and temperature [1-3]. Among those studies, $\mathrm{pH}$-responsive copolymers have been the predominant stimulus-responsive blocks owing to unique property of smart response to convenient $\mathrm{pH}$ change.

pH-responsive double hydrophilic block copolymers can spontaneously self-assemble into two or more types of micelles triggered by hydrophobic interaction, hydrogen-bonding and electrostatic interaction induced by $\mathrm{pH}$ changes [4-7]. The fascinating supramolecular self-assembled micelles have been the most attractive interest as nanocarrier. While the dilemma of constructing stimuli zwitterionic doubles hydrophilic block copolymer is avoiding the complicate and multi-steps preparation process [6-7]. Therefore, more brief and facile methods for fabricating tunably stimuli zwitterionic double hydrophilic block copolymer comprising different responsive segment should be developed.

Herein, a new method was developed for preparing novel PEGylated zwitterionic triblock copolymer via ATRP using protecting group chemistry, followed by one step hydrolysis. The novel PEGylated zwitterionic triblock copolymer contianing polybase 
poly(2-aminoethyl methyl acrylate) PAEMA block and polyacid poly(methacrylic acid) PMAA block is expected to possess remarkable self-assembly behaviors.

\section{Experimental Section}

Materials. Bromide-capped poly-(ethylene glycol) macroinitiator $\left(\mathrm{PEG}_{113}-\mathrm{Br}\right)^{8}$ and $N$-(tert-butoxycarbonyl) amino-ethyl methacrylate $(t \text {-BocAEMA })^{9}$ were prepared according to literature procedures. All other chemicals were purchased from Sigma-Aldrich and used as received.

Preparation of PEG- $\boldsymbol{b}$-PtBocMA-Br Diblock Copolymer. A reaction flask with a magnetic stirrer and a rubber septum was charged with $\mathrm{PEG}_{113}-\mathrm{Br}(2.06 \mathrm{~g}, 0.4 \mathrm{mmol})$, $\mathrm{CuBr}$ (57.4 mg, $0.4 \mathrm{mmol})$, bpy (124.9 mg, $0.8 \mathrm{mmol}), t \mathrm{BocMA}(6.4 \mathrm{ml}, 40 \mathrm{mmol})$ and toluene $(10 \mathrm{~mL})$. The flask was degassed by three freeze-pump-thaw cycles, back-filled with $\mathrm{N}_{2}$, and then placed in an oil bath thermostated at $80{ }^{\circ} \mathrm{C}$ to start the polymerization under a $\mathrm{N}_{2}$ atmosphere. After $2 \mathrm{~h}$, the flask was quenched in the liquid nitrogen, exposed to air, and then diluted with $10 \mathrm{~mL}$ of dichloromethane. The copper catalysts were removed by after passing through a silica gel column. The solvent was then concentrated by a rotary evaporator and precipitated into excess cold n-hexane to remove residual monomers three times. The obtained colorless product was dried in a vacuum oven overnight at room temperature. The yield was $\sim 40 \%$.

Preparation of PEG- $b$-PtBocMA- $b$-PtBocAEMA Triblock Copolymer. The triblock copolymer was synthesized via ATRP method by polymerized $t$ BocAEMA monomer using $\mathrm{PEG}_{113}-b-\mathrm{P} t \mathrm{BocMA}_{42}-\mathrm{Br}$ as the macroinitiator. A typical procedure was as follows: $\mathrm{PEG}_{113}-b-\mathrm{P} t \mathrm{BocMA} 42-\mathrm{Br}(2.0 \mathrm{~g}, 0.2 \mathrm{mmol}), \mathrm{CuBr}(28.7 \mathrm{mg}, 0.2 \mathrm{mmol})$, bpy $(62.5 \mathrm{mg}, 0.4 \mathrm{mmol}), t \operatorname{BocAEMA}(4.6 \mathrm{~g}, 20 \mathrm{mmol})$ and toluene $(10 \mathrm{~mL})$ were charged into a $50 \mathrm{~mL}$ reaction flask. The flask was degassed by three freeze-pump-thaw cycles, back-filled with $\mathrm{N}_{2}$, and then placed in an oil bath thermostated at $80{ }^{\circ} \mathrm{C}$ to start the polymerization under a $\mathrm{N}_{2}$ atmosphere. After $16 \mathrm{~h}$, the reaction flask was quenched in liquid nitrogen, exposed to air, and diluted with $10 \mathrm{~mL}$ of chloroform. After passing though a silica gel column to remove the copper catalysts and concentrating the solvent by a rotary evaporator, the residues were precipitated into excess n-hexane to remove residual monomers. After the supernatant solution was discarded, the product was collected as colorless solid. The prepared sample was then dried in a vacuum oven overnight at room temperature. The overall yield was $\sim 55 \%$.

Hydrolysis of PEG- $b$-P $t$ BocMA- $b$-PtBocAEMA Triblock Copolymer. To remove the $t$-Boc groups, $1.0 \mathrm{~g}$ of PEG- $b$-P $t$ BocMA- $b$-P $t$ BocAEMA was dissolved in 5 $\mathrm{mL}$ of trifluoroacetic acid (TFA) and stirred for $5 \mathrm{~h}$ at room temperature. TFA was then removed by vacuum evaporation, and the residue was rinsed with diethyl ether, filtered, and washed by diethyl ether three times. The colorless product PEG- $b$-PMAA ${ }_{42}-b$-PAEMA 50 was dried overnight in a vacuum oven overnight at room temperature. The overall yield was $\sim 85 \%$.

Characterizations. All ${ }^{1} \mathrm{H}$ NMR spectra were recorded using a Bruker Avance 400 NMR spectrometer with $\mathrm{CDCl}_{3}, \mathrm{D}_{2} \mathrm{O}$ or DMSO- $\mathrm{d}_{6}$ as the solvent and tetramethylsilane as the internal standard. Molecular weights and molecular weight distributions were determined by THF GPC with PS as calibration standard.

\section{Results and Discussion}

Preparation of PEG- $\boldsymbol{b}$-PtBocMA- $\boldsymbol{b}$-PtBocAEMA. ATRP is a versatile method to prepare bock copolymer containing P $t$ BocMA or P $t$ BocAEMA under mild condition $[7$, 10]. The synthesis route as shown in Scheme. 1. 
In order to achieve highly initiator efficiency for the subsequent polymerization, controlling the low conversion by shorten the polymerization of $t$ BocMA time to $2 \mathrm{~h}$. well defined PEG- $b$-P $t$ BocMA-Br diblock copolymer was obtained with low monomer conversion of $42 \%$, the DP was 42 from ${ }^{1} \mathrm{H}$ NMR analysis (as shown Figure 1). In the polymerization of $t$ BocAEMA, $\mathrm{PEG}_{113}-b-\mathrm{P} t \mathrm{BocMA}_{42}-\mathrm{Br}$ was used as macroinitiator. The DP of the PtBocAEMA block was calculated by ${ }^{1} \mathrm{H}$ NMR to be 50 by comparing the intergral ratio of resonance at $3.3 \mathrm{ppm}$ (e) or $4.2 \mathrm{ppm}$ (f) and $3.6 \mathrm{ppm}$ (c), characteristic of PtBocAEMA and PEG blocks, respectively. THF GPC traces (as shown in Figure 2) revealed a narrow and symmetric peak for both the diblock and triblock copolymer with an $M_{\mathrm{n}} 12600$ and $28800 \mathrm{~g} / \mathrm{mol}$, an $M_{\mathrm{w}} / M_{\mathrm{n}}$ of 1.2 and 1.35 , thus denoted as PEG- $b$-P $t$ BocMA ${ }_{42}-\mathrm{Br}$ and $\mathrm{PEG}_{113}-b-\mathrm{P} t \mathrm{BocMA}_{42}-b-\mathrm{P} t$ BocAEMA $_{50}$.

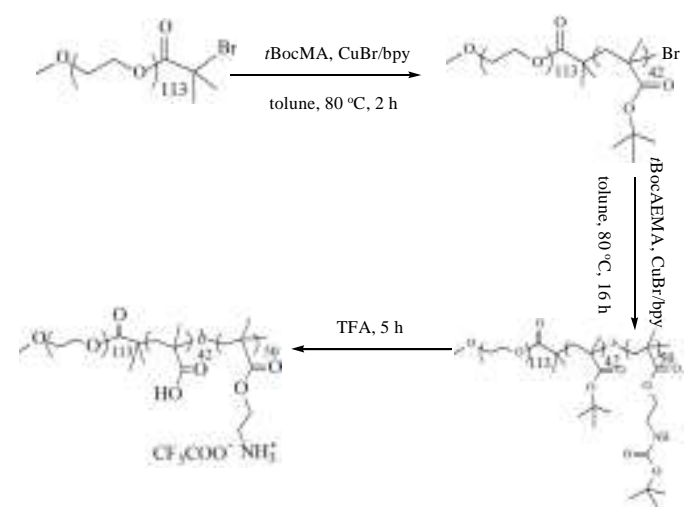

Scheme 1. Synthetic Route for the Preparation of PEG- $b$-PMAA- $b$-PAEMA zwitterionic Triblock Copolymer

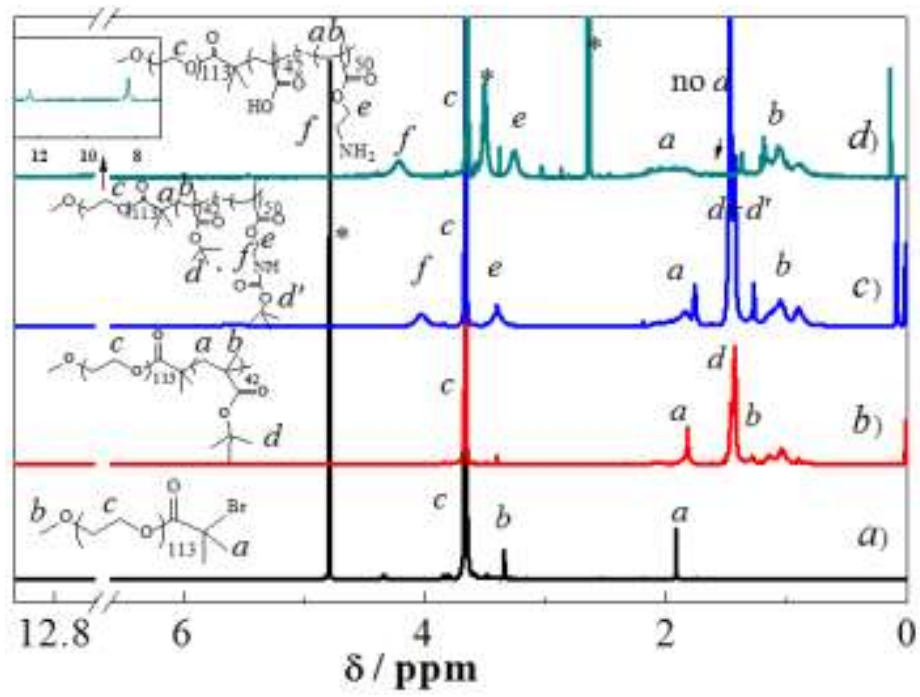

Figure 1. ${ }^{1} \mathrm{H}$ NMR spectra recorded for (a) $\mathrm{PEG}_{113}-\mathrm{Br}\left(\mathrm{D}_{2} \mathrm{O}\right)$, (b) $\mathrm{PEG}_{113}-b$-P $t \mathrm{BocMA}_{42}\left(\mathrm{CDCl}_{3}\right)$, (c) $\mathrm{PEG}_{113}-b-\mathrm{P} t \mathrm{BocMA}_{42}-b-\mathrm{P} t \mathrm{BocAEMA}_{50}\left(\mathrm{CDCl}_{3}\right)$, (d)PEG ${ }_{113}-b-\mathrm{PMAA}_{42}-b-\mathrm{PAEMA}_{50}\left(\mathrm{DMSO}-d^{6}\right)$ 


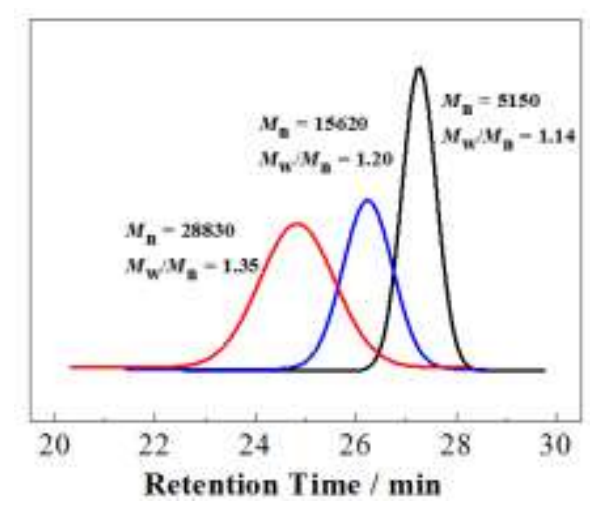

Figure 2. THF GPC traces of (a) $\mathrm{PEG}_{113}-\mathrm{Br}$, (b) $\mathrm{PEG}_{113}-b-\mathrm{P} t \mathrm{BocMA}_{42}$, (c) $\mathrm{PEG}_{113}-b-\mathrm{P}$ BocMA $_{42}-b-\mathrm{P} t$ BocAEMA $_{50}$

Hydrolysis of PEG- $\boldsymbol{b}$-PtBocMA- $\boldsymbol{b}$-PtBocAEMA. After hydrolysis of the obtained triblock copolymer, by ${ }^{1} \mathrm{H}$ NMR spectrum analysis, it is clearly shown that the signal of Boc group at $1.44 \mathrm{ppm}$ was disappeared, indicating the completely hydrolysis of P $t$ BocMA and P $t$ BocAEMA block, moreover, new characteristic signals were observed at $8.3 \mathrm{ppm}$ and $12.3 \mathrm{ppm}$ indicating the $\mathrm{NH}_{2}$ and $\mathrm{COOH}$ group appeared compared to PEG- $b$-P $t$ BocMA- $b$-P $t$ BocAEMA triblock copolymer. By ${ }^{1} \mathrm{H}$ NMR spectrum analysis, after hydrolysis of PEG- $b$-P $t$ BocMA- $b$-P $t$ BocAEMA, PEG block was not to be affected. Thus, the zwitterionic PEG- $b$-PMAA- $b$-PAEMA will retain the structure feature of PEG- $b$-P $t$ BocMA- $b$-P $t$ BocAEMA triblock copolymer, thus denoted as $\mathrm{PEG}_{113}-b$ PMAA $_{42}-b$-PAEMA 50 and the study of supramolecular self-assembly behavior is on going.

\section{Conclusions}

Well defined PEG- $b$-P $t$ BocMA- $b$-P $t$ BocAEMA triblock copolymer, was synthesized via sequential atom transfer radical polymerization (ATRP) in toluene. The obtained triblock copolymer was successfully converted into PEGylated zwitterionic PEG- $b$-PMAA- $b$-PAEMA triblock copolymer by hydrolysis. The PEGylated zwitterionic PEG- $b$-PMAA- $b$-PAEMA triblock copolymer comprising amine based polybase block and $\mathrm{pH}$-sensitive carboxyl group based polyacid block will exhibit remarkable self-assembly behaviors, induced by electrostatic interaction hydrogenbonding interaction, respectively. The synthesis of the $\mathrm{pH}$-responsive zwitterionic PEG- $b$-PMAA- $b$-PAEMA triblock copolymer bearing lager number of amine moieties will provide a light for design of novel anti-cancer drug or DNA delivery carriers.

\section{Acknowledgments}

The authors gratefully acknowledged the financial support of the National Natural Science Foundation of China (No. 21204010, No. 51473035), the Fundamental Research Funds for the Central Universities (No. 2232014D3-35), the Research Program of Shanghai Science and Technology Commission (No. 13NM1400102).

\section{References}

[1] H. Cölfen. Macromol. Rapid Commun. 22 (2001) 219-252.

[2] S. Y. Liu, S. P. Armes. Angew. Chem., Int. Ed. 41 (2002) 1413-1416.

[3] X. Z. Jiang, G. Y. Zhang, R. Narian, S. Y. Liu. Langmuir. 25 (2009) 2046-2054. 
[4] S. Holappa, L. Kantonen, F. M. Winnik, H. Tenhu. Macromolecules. 37(2004) 7008-7018.

[5] V. Bütün, S. Y. Liu, J. V. M. Weaver, X. Bories-Azeau, Y. L. Cai, S. P. Armes. 66 (2006) 157-165.

[6] Y. L. Cai, S. P. Armes. Macromolecules. 37 (2004) 7116-7122.

[7] H. Liu, C. H. Li, H.W. Liu, S. Y. Liu. Langmuir. 25 (2009) 4724-4734.

[8] S. Y. Liu, J. V. M. Weaver, Y. Q. Tang, N. C. Billingham, S. P. Armes.

Macromolecules. 35 (2002) 6121-6131

[9] K. Kuroda, W. F. DeGrado. J. Am. Chem. Soc. 127 (2005) 4128-4129.

[10] W. H. Ji, D. Panu, R. Noelle Palumbo, R.P. Tang, C. Wang. Biomacromolecules. 12 (2011) 4373-4385 